\title{
Study on Reliability Optimization Algorithm of Computer Network Linlin Wang ${ }^{1, a}$

\author{
${ }^{1}$ Zhongshan Torch Polytechnic, Zhongshan, Guangdong, 528436 \\ ${ }^{a}$ email
}

Keywords: Reliability Optimization, Computer Network, Algorithm

\begin{abstract}
With the rapid development of computer technology, computer networks are gradually emerging and penetrate into all areas of the national economy, regardless of the political and economic or financial and commercial, more and more inseparable from all sectors of computer networks. Once the computer network failure, the various sectors of the national economy will have a significant impact. The country's chaos and political turmoil, so the reliability of the computer network is also increasingly high demands on the reliability of computer network research. All of those have very huge economic and social benefits.
\end{abstract}

\section{Introduction}

After the 20th century, 90 years, the increasing use of computers around the world, from the beginning of the Office of the communication between several computers, to the entire unit of computer communication, until a computer access to the Internet throughout the country, So as to achieve information and software sharing. Computer network to the social production and people's lives to bring great convenience, due to some of its own characteristics, in the event of network failure, will result in huge losses, or even irreparable damage. For the enterprise, once the internal network failure, it will cause millions of dollars in losses. For banks, the problem is more serious, authoritative statistics, the computer network paralyzed for 1 hour, will cause 6 million US dollars in losses, if paralyzed more than a day, then the bank completely bankrupt. For securities institutions, if the network problems, then the Internet users will cause huge economic losses. Now power, transportation, finance and other industries are connected to the Internet, once a large area of the network paralysis and will cause the various sectors of the national economy catastrophic impact, and this effect will spread to ordinary people's daily life, Thus causing social unrest, to national security and stability. It is precisely because the computer network for the national economy, national security and even people's daily lives have such a significant impact, it is necessary to continue through optimization and design, and vigorously improve the reliability of computer networks, as much as possible to reduce their failure probability.

\section{Related Theory of Computer Network Reliability}

Computer network refers to the network through the communication link, interactive devices and related network protocols, will be distributed in different regions of the mutual independence of several computers connected to each other, so that it can complete a specific function of the complex network system. In this network, any computer can not complete control of other computers each computer has its own independence. But these computers are not unrelated, you can access through the computer network free of information resources. The communication sub-network and the user resource sub-network together constitute a computer network, the former is responsible for the information transfer between computers, which is responsible for processing resource information, and provided to the computer network. Image, the computer network is independent of the computer in the middle of a bridge between the blocks, so that it can freely exchange of information.

The inherent structure and specific functions of a computer network make it unique. First, the computer network there are a lot of computers, these computers are often located far away, there is a large geographical gap; Second, in the computer network, although you can share resources, but in 
fact each computer has a larger Third, the computer network in the computer to achieve interconnection, can not be separated by the transmission switching equipment and communication links composed of communication facilities, so that the computer network is not independent of the existence of the computer network is not independent of each other, ; Fourth, different types of computer network communication and interoperability of computer systems, the computer network has put forward a higher level of requirements, the need for computer networks through communication facilities to achieve collaborative processing and resource sharing interoperability.

After a long period of development, scholars of various countries on the computer network reliability measurement are discussed, in general can be summarized and summarized as: computer network connectivity, the survival of computer networks, computer networks, anti-destructive and in a variety of models under the computer network components of the effectiveness. Among the current reliability research areas, the most extensive is the computer network connectivity. This is because the components of the computer network and the basic node in the network work properly, can provide reliable links for each user. Reliability is an important measure of computer network connectivity standards. Computer network reliability refers to the computer network in the specified time, network connectivity state, under certain conditions, the computer network to complete the required function of the probability, that is able to meet the communication requirements of computer network design and planning is one of the important parameters . At the same time, whether the computer network operating capacity in the normal range, but also by its own topology determined by the structure.

\section{Computer Network Reliability Model}

Practical operation, the graphical system is the most easy to deconstruct, the usual approach is to first complete the object system modeled network system to achieve. Especially for complex systems research, more clearly and accurately, such as in the logistics network, transmission and distribution system network and communication networks and other fields have been mature applications. Computer network reliability problem can also be modeled as a graph of the reliability problem, you can use probability graph $G(v, e)$ to represent ( $v$ - computer network host, server or user terminal, e-computer network link). The normal running state of the nodes and the normal running state of each side of the graph are given certain probability values, and finally the probability graph of the computer model is obtained. In general, the reliability of graph mainly includes two aspects of analysis and design. The former is a given graph of the reliability of the corresponding calculation, and then design the corresponding graph, this map to maximize the reliability, Provided that the relevant parameters have been established. Often can not directly solve the reliability of the map, then you can proceed from the opposite of reliability, first seeking the degree of failure, and then come to the reliability.

The traditional computer network reliability algorithm, in its essence, is to simplify the computer network model using graph theory, and then through numerical analysis, probability statistics to calculate, and ultimately solve. The complete state enumeration method, the non-intersection algorithm, the tolerant rejection principle algorithm, the factor decomposition algorithm, the special network and so on belong to the computer network reliability traditional algorithm. The complete state enumeration method is widely used in the computer network reliability because of its simple method. This method fully enumerates the state of the computer network links and nodes, and all the state of the corresponding check to determine the appropriate elements to ensure that the computer network to normal operation. Non-intersection and algorithm need to have a premise, that is known to meet the normal operation of computer networks, the smallest part of the collection, and already know the reliability of computer network components. After satisfying these two conditions, the reliability of the computer network can be calculated by the disjoint and algorithm. Non-intersection and algorithm mainly includes two aspects, on the one hand through the number of theory, graph theory to find the normal operation of the computer network to meet the minimum components required for the collection; the other is the normal operation of the computer network components required to normal minimum The probability of running the calculation, mainly 
through the probability of statistical methods to achieve. It can be seen that the probability of normal operation of all the network components in the minimum set of components required maintain the computer network is the core of the non-intersection and algorithm.

\section{The Design Principle of Reliability of Computer Network and the Optimum Design}

Computer networks can be simplified according to their characteristics into a node and link set, the benefits of this can be its theoretical analysis of the math. The corresponding mathematical model is established by using the theory of queuing theory and graph theory, and the theoretical analysis and calculation of the computer network are carried out. The current computer network mainly has the following mathematical models: two and a variety of work model, failure independent and dependent model, single threshold and multi-threshold model, hardware and software reliability model.

Assuming that there are two or more modes of operation of the network components, it is assumed that the components of the two modes of operation are either active or inactive, and that multiple modes of operation take into account a variety of operating and failure states State, in which the performance of network components gradually weakened, with a variety of different modes of operation. Assume that the middle state of the network component is not full capacity, or 0 capacity, but the capacity gradually changes. It has also been assumed that the working state of the network and components varies between failures and normal, since the modes of operation of the network elements are the same or different and the intermediate states are discrete. In practice, the similarity between multiple operating modes and actual conditions is greater, but in reality, the number of states is increasing, which makes the calculation more and more difficult.

The premise of the former is to assume that the failure of each component is independent of each other, while the latter is the premise of the establishment of the opposite, the failure of network components are interrelated. In practice, there is a link between most components, so the latter is more widely used in practice. Dependency model is the biggest feature is the need for state enumeration, in order to reduce the number of enumerations can be used to calculate the corresponding approximation. In some special cases, the dependence of network failure mode will greatly reduce the number, so that the reliability of the analysis algorithm is greatly reduced. Although the failure of independent and actual situation there is a big difference, but to a large extent facilitate the establishment of the model, easy to carry out research.

Genetic algorithm is based on the biologist Darwin's biological evolution theory of genetics and natural selection theory and simulation of a complete set of computational models to search for the optimal solution of the system solution. The two biggest advantages of this approach are: spatial search global solution and parallelism implicit, and its solution is not subject to any constraints of constraints, while coding technology has not complex genetic operations. In order to obtain a relatively optimal or optimal solution to an object problem, GA maps the image of the deconstruction process of the object problem into the natural evolution of "chromosomes" in biology. This pioneering way of thinking, is drawing on the natural evolution process of randomness, parallel precision and automatic matching and other advantages, optimization of individual in order to highlight from the group, will take a series of steps (copy, crossover, mutation) To achieve sustained development and improvement. At present, GA is widely used in many applications, such as: image recognition, optimization design, model optimization, genetic processing and machine control and so on. People's attention to GA technology is dependent on the rapid progress of computer technology.

\section{Conclusion}

The rapid development of computer network, on the one hand promotes the reliability of computer network factors increasing, on the other hand, the reliability of computer network system has put forward higher requirements. Therefore, the reliability factors of computer network and the reliability index system of computer network mentioned in this paper need to be improved, enriched 
and improved. The computer network link cost model and the computer network reliability model should also take into account the stochastic nature, uncertainty and the objective function of the human factors in the actual operation of the computer network.

\section{References}

[1] Liu Lu. Computer network reliability optimization technology [J]. Oriental corporate culture, $2010(02)$

[2] Zhang Zhi, Xu Wei. On the computer network reliability optimization design [J]. Science and Technology Information, 2006 (35)

[3] Deng Zhiping.Computer network reliability optimization design [J]. Technology Plaza, 2010 (01)

[4] Liang Lv. Computer network reliability of the factors and countermeasures [J]. Private Science and Technology, 2011 (01)

[5] Luo Jingfeng, Liu Yanqiu. Application of intelligent algorithm in reliability design of all-terminal network [J]. Computer Measurement \& Control, 2008 (02) 